\title{
Simultaneous Biliary Drainage and Portal Vein Embolization Before Extended Hepatectomy for Hilar Cholangiocarcinoma: Preliminary Experience
}

\author{
Boris Guiu • Pierre Bize • Nicolas Demartines • \\ Mickaël Lesurtel • Alban Denys
}

Received: 14 May 2013/Accepted: 2 June 2013/Published online: 11 July 2013

(C) Springer Science+Business Media New York and the Cardiovascular and Interventional Radiological Society of Europe (CIRSE) 2013

\begin{abstract}
Background Patients with resectable hilar cholangiocarcinoma often present obstructive jaundice and a small future remnant liver (FRL) ratio. A sequential approach comprising preoperative biliary drainage followed by portal vein embolization (PVE) is usually performed but leads to long preoperative management (6-12 weeks) before patients can undergo resection. To simplify and shorten this phase of liver preparation, we developed a new preoperative approach that involves percutaneous biliary drainage and PVE during the same procedure. We report the outcomes of this combined procedure.

Methods During 1 year, four patients underwent simultaneous biliary drainage and PVE followed 1 month later by surgical resection of hilar cholangiocarcinoma. Liver volumes were assessed by CT before, and 1, and 3 months after the combined procedure. Serum liver enzymes were assessed before and 1 month after the combined procedure. Results The combined procedure was feasible in all cases, with no related complications. After the combined procedure, transaminases remained stable or decreased, whereas gamma-glutamyl-transpeptidase, alkaline phosphatase, and
\end{abstract}

B. Guiu $(\bowtie) \cdot$ P. Bize · A. Denys

Digestive \& Oncologic Imaging and Interventional Radiology unit, Department of Radiology and Interventional Radiology, CHUV University Hospital, Rue du Bugnon 46, 1011 Lausanne, Switzerland

e-mail: boris.guiu@chu-dijon.fr

\section{N. Demartines}

Department of Visceral Surgery, Centre Hospitalo-Univeristaire Vaudois, Lausanne, Switzerland

M. Lesurtel

Department of Surgery, University Hospital Zürich, Zurich, Switzerland bilirubin decreased. During the first month, the left lobe volume increased by $+27.9 \%$ (range $19-40.9 \%$ ). The FRL ratio increased from 24.9 to $33.2 \%$. All patients underwent $\mathrm{R} 0$ liver resection with a favorable postoperative outcome. The remnant liver volume increased by $+132 \%$ (range 78-245\%) between 1 and 3 months.

Conclusions Simultaneous percutaneous biliary drainage and PVE is feasible. This all-in-one preoperative approach greatly decreases waiting time until surgical resection. These encouraging results warrant further investigation to confirm the safety and to evaluate the reduction in the dropout rate for liver resection in this tumor with poor prognosis.

Keywords Portal vein - Embolization - Biliary tract . Drainage $\cdot$ Bile obstruction $\cdot$ Liver regeneration

\section{Introduction}

Surgery for hilar cholangiocarcinoma is a complex procedure usually requiring extended hepatectomy, resection of the caudate lobe, extrahepatic bile duct resection, and biliary-enteric anastomosis. In-hospital mortality after hepatic resection is usually higher than $10 \%$ (up to $21 \%$ ) in patients with obstructive jaundice, whereas it is $8 \%$ in cirrhotic patients and only $1 \%$ in patients with normal liver [1, 2]. Biliary obstruction can result in bacterial translocation, hemostasis impairment, malnutrition, renal insufficiency, and an increased risk of postoperative liver dysfunction [3, 4]. In this context, major surgical resection is risky and most surgical teams recommend performing preoperative biliary drainage [5-8].

The future remnant liver (FRL) volume is another challenge to bring the patient to surgery because it often 
is insufficient given the large volume of resected liver in extended hepatectomy for hilar cholangiocarcinoma. In this setting, it is generally accepted that there is a high risk of postoperative hepatic failure when the FRL volume is lower than $40 \%[4,5,9]$. Portal vein embolization (PVE) has been proposed to increase the functional reserve of the remnant liver [10-12]. The usual approach is first to drain the biliary tree of the FRL until bilirubin has fallen below $5 \mathrm{mg} / \mathrm{dl}$ and then to perform contralateral PVE. However, this preoperative management takes time before the patient can be resected. Indeed, it usually takes more than 4 weeks for the jaundice to resolve [5] and an additional 4 weeks to obtain sufficient liver regeneration after the PVE. During this period, tumor progression may occur thereby precluding any curative treatment.

To simplify and shorten this phase of liver preparation, we developed a combined preoperative approach in which percutaneous biliary drainage and PVE are performed during the same procedure. The aim of this study is to report the outcomes of this combined approach used in four patients with hilar cholangiocarcinoma.

\section{Materials and Methods}

This study consisted of a retrospective analysis of our experience with combined biliary drainage and portal vein embolization performed during the same procedure before surgical resection of hilar cholangiocarcinoma. All patients gave their written informed consent for the procedure and our institutional review board approved the retrospective analysis of their data.

During a 1-year period, 12 patients were referred to our department for percutaneous biliary drainage for hilar cholangiocarcinoma that was either histologically proven or highly suspected on baseline imaging data. Among them, four patients were considered eligible for surgical resection but required a preoperative PVE procedure, because the planned resection would have left less than $40 \%$ of functional liver parenchyma estimated by CT liver volumetry. The therapeutic strategy was discussed at our weekly liver tumor board meeting, including liver surgeons, oncologists, hepatologists, and interventional radiologists. These four patients were considered for a combined (biliary drainage + PVE) procedure and thus were included in this retrospective study. Three men (age range: 57-71 years) and one woman (age: 57 years) had hilar cholangiocarcinoma classified as Bismuth-Corlette stage II $(n=1)$ and IIIA $(n=3)$. None of the patients had liver cirrhosis or had undergone prior therapy, such as systemic chemotherapy. No patient had clinical signs of sepsis or cholangitis.

\section{Technique}

Combined biliary drainage and PVE were performed under general anesthesia (Fig. 1). All patients received
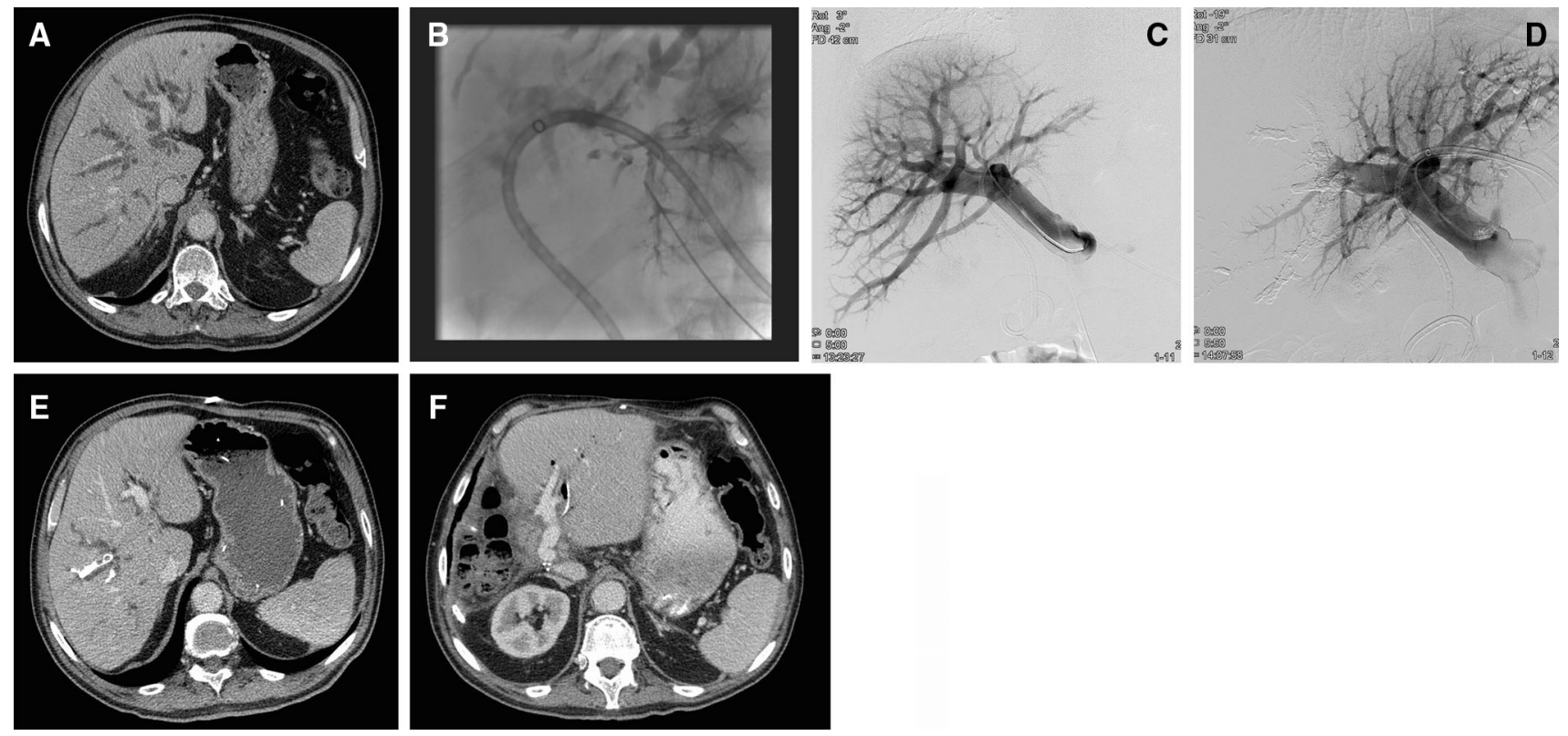

Fig. 1 Axial CT scan (A) in a 71-year old patient showing biliary obstruction due to hilar cholangiocarcinoma (Bismuth-Corlette IIIa). Puncture of the portal venous system just after internal-external biliary drainage of the FRL (B). Portography before (C) and after
(D) PVE using NBCA. Axial CT scan (E) performed 3 weeks later, showing hypertrophy of the left lobe (19\% increase). Axial CT scan (F) performed 2 months after extended hepatectomy showing major hypertrophy of the remnant liver (78\% increase) 
intravenous prophylactic antibiotics (ceftriaxone). For biliary access, we used a "1-stick" technique [13] using a Neff introducer set (Cook Medical, Bloomington, IN). First, the left bile ducts (preferentially a segment III branch) were punctured under US guidance with a $15-\mathrm{cm}-$ long, 21-gauge Chiba introducer needle and opacified. Then, a 0.018-inch Cope mandril guidewire was introduced through the needle to gain initial access. Finally, the triaxial dilator and sheath combination was introduced over the micro-guidewire allowing accommodation of a 0.035 inch guidewire in the outer sheath after removal of the inner dilator and sheath. A 11-cm-long, 8F sheath (Super Arrow Flex PSI set, Teleflex Medicine, Athlone, Ireland) was inserted into the bile ducts to secure access and facilitate the passage of a $5 \mathrm{~F}$ shaped catheter (Soft-vu Berenstein, AngioDynamics, Cambridge, UK) and a stiff hydrophilic 0.035-inch guidewire (Terumo, Tokyo, Japan) used to cross the biliary obstruction and ultimately gain access to the duodenum. Then, a 0.035-inch, 80-cm Amplatz extra-stiff wire (Cook Medical) was inserted to allow the passage of an $8.5 \mathrm{~F}$ internal-external drainage catheter (Mac-Loc, Cook Medical), positioned so that the side holes would drain the left intrahepatic ducts and the "pigtail" configuration of the distal end of the catheter will be placed into the duodenum. If the primary biliary drainage was technically successful with no evidence of hemobilia, the PVE procedure was performed immediately thereafter.

Under US guidance, a left portal vein branch (preferentially the portal branch corresponding to the bile duct punctured for biliary drainage) was accessed using the same type of instruments as for the biliary access (21-gauge Chiba needle, Neff introducer set). If the US examination was obscured by artifacts resulting from the biliary procedure, the portal vein branch was punctured under fluoroscopic guidance, close to the previously punctured bile duct. A 5F shaped catheter (Soft-vu Berenstein) was advanced over a 0.035-inch hydrophilic guidewire (Terumo, Tokyo, Japan) into the portal trunk where portography was performed. The PVE procedure was performed as described elsewhere [11, 14]; the right second-order portal branches were selectively catheterized one by one using the shaped catheter and then embolized with boluses of $0.5 \mathrm{ml}$ of a mixture of Lipiodol and n-butyl-cyanoacrylate (Histoacryl, B Braun, Tuttlingen, Germany) after checking that the flow was hepatopetal (a mixture of $1 \mathrm{ml}$ of $n$-butyl cyanoacrylate with $1-3 \mathrm{ml}$ of iodized oil was used). Segment IV was not embolized. Following embolization, the shaped catheter was carefully flushed into the right portal branch with $5 \%$ glucose solution and then repositioned in the portal trunk, where post-PVE portography was performed. During the 2 days following the procedure, pain was managed using morphine titration when necessary.
Volumetric CT Assessment and Outcome Evaluation

Baseline computed tomography (CT) imaging data were obtained a mean 12 (range: 1-26) days before the combined procedure, and follow-up imaging was performed approximately 1 month (mean: 26 days; range: 22-33 days) and 3 months (mean: 92 days; range: 80-105 days) after the combined procedure. CT images with a $2.5-\mathrm{mm}$ slice thickness were obtained during the portal phase $(70 \mathrm{~s}$ after injection of $100 \mathrm{cc}$ of $350 \mathrm{mg} / \mathrm{ml}$ iodine at $3 \mathrm{cc} / \mathrm{s}$ ) after injection of contrast media with a 64-detector row Lightspeed multislice CT unit (General Electric, Milwaukee, WI). On each image, the whole liver and the left lobe (segments II and III) were delineated with a hand-held cursor and their respective volumes were automatically calculated by the workstation (Advantage windows, General Electric). The ratio between the left lobe volume and the whole liver volume was then calculated and defined as the FRL ratio.

\section{Liver Enzymes}

Serum liver enzymes were assessed by measuring serum aspartate aminotransferase (AST), serum alanine aminotransferase (ALT), gamma-glutamyl-transpeptidase (GGT), alkaline phosphatase (ALP), prothrombin time (PT), and total bilirubin levels before and 1 month after the combined procedure.

\section{Statistical Analysis}

Categorical variables were described using percentages. The levels of liver enzymes and liver volumes were expressed as means and standard deviations. Given the low statistical power due to the small number of patients, comparisons of laboratory tests and liver volumes were not performed. All descriptive analyses were performed using Stata software version 11.0 (Stata Corporation, College Station, TX).

\section{Results}

The mean baseline bilirubin level was $136 \mu \mathrm{mol} / 1$ (standard deviation: 114). The combined procedure (biliary drainage + PVE) was feasible in all cases. No complications related to the procedure occurred. After the combined procedure, AST and ALT remained stable or decreased, whereas GGT, ALP, and bilirubin decreased and PT tended to decrease (Table 1).

Regarding liver volumes during the first month (Table 2), the left lobe (segment II + III) increased in size from 550 to $692 \mathrm{ml}$ (mean increase: $27.9 \%$ ), whereas total 


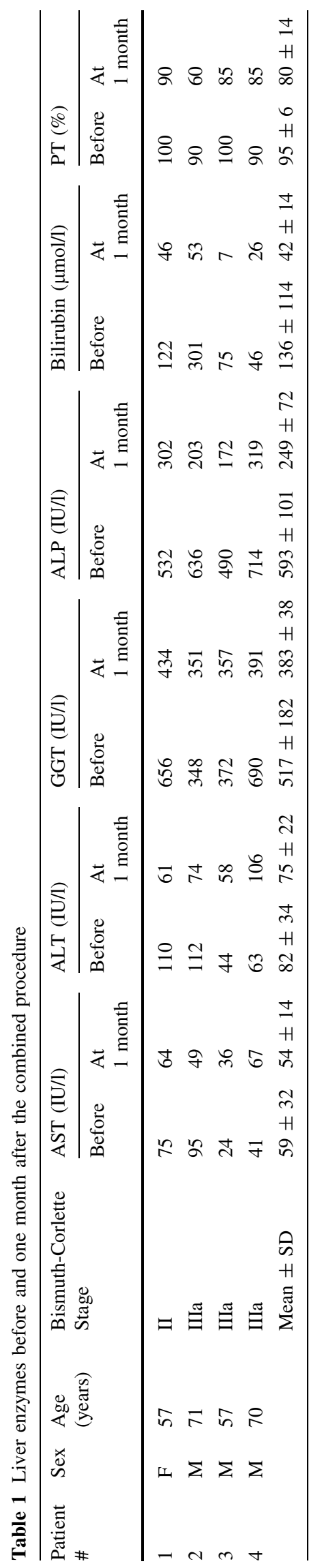

liver volume decreased from 2,178 to 2,046 $\mathrm{ml}$ (mean decrease: $6 \%$ ). The FRL ratio increased from 24.9 to $33.2 \%$, leading to an $8.3 \%$ increase. Patients were operated on the day following the CT-scan evaluation at 1 month. All patients underwent R0 right hepatectomy extended to segment IV and I, which pathological examination confirmed the diagnosis of hilar cholangiocarcinoma. The mean duration of the surgical procedure was 398 (range: 324-445) min. The mean transfusion requirements were 1.75 (range: 0-4) units, and the mean patient stay in an intensive care unit was 4 (range: 0-9) days. Postoperatively, patient \#2 developed a fistula from the biliary-enteric anastomosis which was managed conservatively. Patient \#3 developed a wound infection, and patient \#4 had a biloma drained percutaneously without subsequent biliary fistula. All of the patients had favorable postoperative outcomes (mean hospital stay: 18 days; range: 11-28 days). At 3 months, the mean remnant liver volume was $1,565 \mathrm{ml}$, leading to a $+132 \%$ mean increase (range: 78-245\%) between 1 and 3 months (Fig. 2).

\section{Discussion}

To our knowledge, this is the first report of such a strategy for the management of resectable hilar cholangiocarcinoma. This technique was successfully applied in our four patients with no related complications, thereby demonstrating its feasibility. In Klatskin tumors, liver resection is the only treatment that allows prolonged survival. Surgery is considered high risk, because it removes for oncologic reasons not only a hemiliver (generally right) but also segments IV and I and requires biliary-enteric anastomosis. Most patients present with obstructive jaundice and a small FRL ratio. In order to avoid postoperative liver failure, a sequential approach that consists of preoperative biliary drainage followed by portal vein embolization is usually performed [3, 5, 6, 9, 15-19]. Biliary drainage (preferentially internal $[8,9,20])$ is performed to relieve the jaundice and thereby prevent cholangitis, multiorgan failure, and impaired hepatic regeneration after surgery [3, 4]. However after biliary drainage, 4 to 6 weeks are needed to reach a bilirubin level below $2-5 \mathrm{mg} / \mathrm{dl}(34-85 \mu \mathrm{mol} / \mathrm{l})$ $[5,9,18,21,22]$ before patients can benefit from PVE. An additional 2-6 weeks is then necessary after PVE to induce a sufficient functional increase in the FRL. Therefore, this approach usually leads to a long preoperative management (6-12 weeks) before patients undergo resection. In a large series of 494 patients managed by biliary drainage followed by PVE, $25 \%$ did not undergo subsequent hepatectomy due to disease progression during the preoperative period [17]. We therefore developed an aggressive preoperative approach by combining biliary drainage and PVE 
Table 2 Liver volumes before and 1 month after the combined procedure

\begin{tabular}{|c|c|c|c|c|c|c|c|c|}
\hline \multirow[t]{2}{*}{ Patient \# } & \multicolumn{3}{|c|}{ FRL volume (ml) } & \multicolumn{2}{|c|}{ Total liver volume } & \multicolumn{3}{|c|}{ Ratio of FRL (\%) } \\
\hline & Before & At 1 month & $\%$ increase & Before & At 1 month & Before & At 1 month & $\%$ increase \\
\hline 1 & 386 & 511 & 32.4 & 1,750 & 1,620 & 22.1 & 31.5 & 9.4 \\
\hline 2 & 424 & 504 & 19 & 2,336 & 1,998 & 18.1 & 25.2 & 7.1 \\
\hline 3 & 930 & 1,108 & 19.1 & 2,373 & 2,376 & 39.1 & 46.6 & 7.5 \\
\hline 4 & 459 & 647 & 40.9 & 2,251 & 2,190 & 20.4 & 29.5 & 9.1 \\
\hline Mean $\pm \mathrm{SD}$ & $550 \pm 255$ & $692 \pm 285$ & $27.9 \pm 10.7$ & $2,178 \pm 290$ & $2,046 \pm 323$ & $24.9 \pm 9.6$ & $33.2 \pm 9.3$ & $8.3 \pm 1.1$ \\
\hline
\end{tabular}

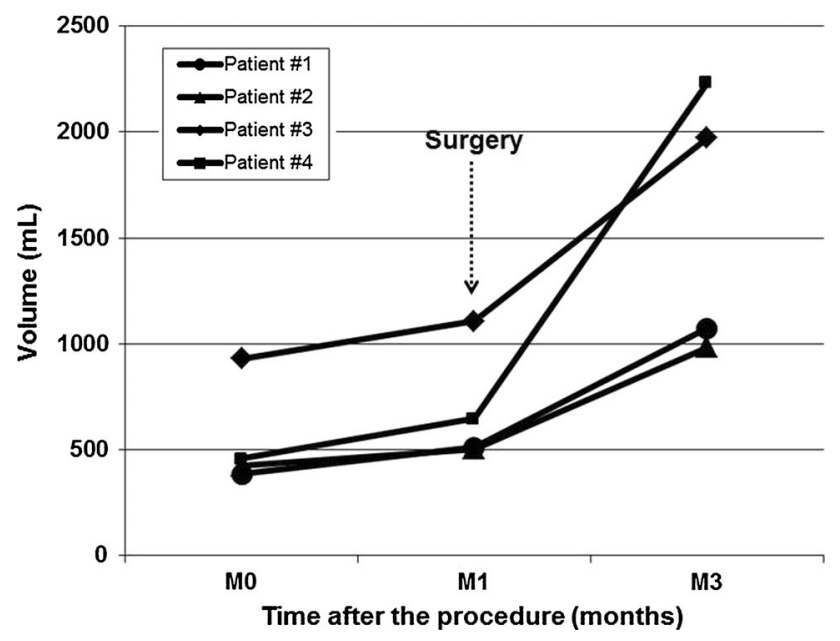

Fig. 2 Changes in volumes of segment II + III for 3 months after the combined procedure (patients were operated on at 1 month)

during the same procedure, to decrease the delay before surgery and to simplify the preoperative management.

The incidence of tumor seeding along the biliary drain tract has been reported as high as $5.2 \%$ and is related to the duration of drainage (the risk of tract recurrence was 3.16 times higher when biliary drainage lasted $>60$ days) [23]. Reducing the duration of biliary drainage therefore also may reduce the risk of tumor seeding.

Cholestasis has been shown to impair hepatic regenerative capacity [24]. The portal triad (portal vein, hepatic artery, and bile duct) is limited to a small anatomic space (the so-called Mall's space), because it is surrounded by the connective tissue sheath of the Glisson capsule [24, 25]. In cases of biliary dilatation, portal vessels are compressed significantly due to their low intravascular pressure (compared with hepatic arteries). This results in decreased portal venous flow and thus impairs hepatic regeneration and hypertrophy after PVE [24, 26]. Interestingly, biliary drainage quickly decreases pressure in bile ducts and thus subsequent portal compression [27], whereas PVE significantly increases the portal flow velocity, which correlates with liver hypertrophy. It has even been reported that PVE could relieve persistent jaundice despite complete biliary drainage by inducing functional enhancement of the nonembolized liver [28]. In a model of cholestatic rat liver [29], the activity of DNA polymerase as a marker of hepatocyte proliferation significantly increased after portal vein ligation whether biliary decompression was performed before or at the same time as portal vein ligation.

Finally, many investigators reported that cholestasis alters the metabolic function of hepatocytes, especially mitochondrial respiratory function [7, 28, 29]. Internal biliary drainage slowly improves mitochondrial activity $[15,20]$, but interestingly, PVE has been shown to increase mitochondrial enzymatic activities as quickly as 2 days after the procedure [30]. All of this strongly suggests that early PVE might benefit icteric patients [29].

After the combined procedure, liver enzymes showed similar evolution to that observed after biliary drainage alone [21]. Indeed, bilirubin level exhibited a marked decrease, as did ALP and GGT [21]. Of note, the relatively low baseline level of bilirubin testifies to the fact that jaundice was probably not long-lasting in our patients. ALT and AST remained stable or decreased, as it is usually observed after PVE alone. When comparing liver volumes during the first month, FLR volume exhibited a mean increase of $27.9 \%$ resulting in an $8.3 \%$ increase of the FLR ratio (FRL/total liver volume). As previously reported [11], it is difficult to compare volume increases with those in other series because of differences regarding: (1) the embolizing agents used, (2) case mixes of liver tumors, (3) time to volumetric assessment, and (4) methods to measure FRL hypertrophy. Although different embolizing materials were used in published series reporting on biliary drainage followed by PVE, volumetric results seem similar. In 189 patients, Nagino et al. [16] reported a $27.4 \%$ increase in FRL volume and a $10 \%$ increase in FRL ratio. In another study, Hong et al. [19] reported a $27.2 \%$ increase in FRL volume with a $7.3 \%$ increase in FRL ratio. However, in a very recent study, the same technique (with same embolic material) applied in noncholestatic livers resulted in a $74 \%$ increase in FRL volume [11]. This could fit with less effective PVE in cholestatic livers. But interestingly, a significant increase in total liver volume has been reported in rat models due to liver swelling in cases of biliary obstruction [31, 32]. Thus, our measurements took into 
account not only volumetric changes related to PVE (i.e., increase) but also those related to biliary drainage (i.e., decrease). This can explain why total liver volume decreased in our four patients, whereas it did not vary in the studies by Nagino et al. and Hong et al. [16, 19]. More importantly and for the same reason, it also is likely that FRL hypertrophy was underestimated in our four patients compared with the preceding studies. After major hepatectomy, liver remnant volumes usually increase from 30 to $50 \%$ during the first 3-6 months [33, 34]. Surprisingly, the volume increase in the left lobe (segment II + III) was much greater between 1 and 3 months $(+132 \%)$, thereby demonstrating the excellent regenerative ability of the liver 1 month after the combined procedure.

Indocyanine green clearance of the FRL or galactosyl human serum albumin scintigraphy are more appropriate methods than liver volumetry to assess the gain in liver function [9]. In addition, these techniques also could be very helpful to determine the optimal waiting time for liver regeneration after the combined procedure. Unfortunately, because of the retrospective design, neither measurement was available in our study. In patients with jaundice due to biliary tract cancer, it has been reported that FRL hypertrophy could be observed as early as 11 days after PVE [15]. Whether or not the synergistic effects of combined biliary drainage and PVE are confirmed, it would be possible to reduce the waiting time before surgery to less than 4 weeks.

\section{Conclusions}

Despite the small number of patients, we have demonstrated the feasibility of concomitant biliary drainage and PVE. It is not reasonable to perform this procedure in cases of sepsis, cholangitis, or if any complications are encountered during the biliary drainage. This all-in-one preoperative approach strongly decreases waiting time until surgical resection, thereby reducing the risk of cancer progression and the risk of tumor seeding along the biliary drain tract. These encouraging results warrant further investigations to confirm the safety of this procedure and to evaluate the reduction in the dropout rate for liver resection in this tumor with poor prognosis. FRL hypertrophy seems comparable to that reported after the classic sequential approach and is probably underestimated. Further studies that evaluate liver function more precisely could demonstrate a synergistic effect of PVE combined with biliary drainage and determine the optimal delay before resection.

Acknowledgments The authors thank Philip Bastable for revising the english. The first author thanks the Société Française de Radiologie, Ligue contre le cancer de Côte d'Or, Laboratoires Roche, and Cardiovascular and Interventional Society of Europe for their support.
Conflict of Interest Boris Guiu, Pierre Bize, Nicolas Demartines, Mickaël Lesurtel, and Alban Denys have no conflict of interest to disclose.

Financial Support No.

\section{References}

1. Belghiti J, Hiramatsu K, Benoist S, Massault P, Sauvanet A, Farges O (2000) Seven hundred forty-seven hepatectomies in the 1990s: an update to evaluate the actual risk of liver resection. J Am Coll Surg 191(1):38-46

2. Iacono C, Ruzzenente A, Campagnaro T, Bortolasi L, Valdegamberi A, Guglielmi A (2013) Role of preoperative biliary drainage in jaundiced patients who are candidates for pancreatoduodenectomy or hepatic resection: highlights and drawbacks. Ann Surg 257(2):191-204

3. Hemming AW, Reed AI, Fujita S, Foley DP, Howard RJ (2005) Surgical management of hilar cholangiocarcinoma. Ann Surg 241(5):693-699 discussion 699-702

4. Oussoultzoglou E, Jaeck D (2008) Patient preparation before surgery for cholangiocarcinoma. HPB (Oxford) 10(3):150-153

5. Belghiti J, Ogata S (2005) Preoperative optimization of the liver for resection in patients with hilar cholangiocarcinoma. HPB (Oxford) 7(4):252-253

6. Nimura Y, Kamiya J, Kondo S et al (2000) Aggressive preoperative management and extended surgery for hilar cholangiocarcinoma: nagoya experience. J Hepatobiliary Pancreat Surg 7(2):155-162

7. Maguchi H, Takahashi K, Katanuma A et al (2007) Preoperative biliary drainage for hilar cholangiocarcinoma. J Hepatobiliary Pancreat Surg 14(5):441-446

8. Nimura Y (2008) Preoperative biliary drainage before resection for cholangiocarcinoma (Pro). HPB (Oxford) 10(2):130-133

9. Yokoyama Y, Nagino M, Nishio H, Ebata T, Igami T, Nimura Y (2007) Recent advances in the treatment of hilar cholangiocarcinoma: portal vein embolization. J Hepatobiliary Pancreat Surg 14(5):447-454

10. Denys A, Prior J, Bize P et al (2012) Portal vein embolization: what do we know? Cardiovasc Intervent Radiol 35(5):999-1008

11. Guiu B, Bize P, Gunthern D, Demartines N, Halkic N, Denys A (2013) Portal vein embolization before right hepatectomy: improved results using n-butyl-cyanoacrylate compared to microparticles plus coils. Cardiovasc Intervent Radiol

12. van Lienden KP, van den Esschert JW, de Graaf W et al (2012) Portal vein embolization before liver resection: a systematic review. Cardiovasc Intervent Radiol

13. Pomerantz BJ (2009) Biliary tract interventions. Tech Vasc Interv Radiol 12(2):162-170

14. De Baere T, Roche A, Elias D, Lasser P, Lagrange C, Bousson V (1996) Preoperative portal vein embolization for extension of hepatectomy indications. Hepatology 24:1386-1391

15. Nagino M, Nimura Y, Kamiya J et al (1995) Changes in hepatic lobe volume in biliary tract cancer patients after right portal vein embolization. Hepatology 21(2):434-439

16. Nagino M, Kamiya J, Nishio $H$, Ebata $T$, Arai $T$, Nimura $Y$ (2006) Two hundred forty consecutive portal vein embolizations before extended hepatectomy for biliary cancer: surgical outcome and long-term follow-up. Ann Surg 243(3):364-372

17. Ebata T, Yokoyama Y, Igami T, Sugawara G, Takahashi Y, Nagino M (2012) Portal vein embolization before extended hepatectomy for biliary cancer: current technique and review of 494 consecutive embolizations. Dig Surg 29(1):23-29 
18. Anaya DA, Blazer DG, Abdalla EK (2008) Strategies for resection using portal vein embolization: hepatocellular carcinoma and hilar cholangiocarcinoma. Semin Intervent Radiol 25(2):110-122

19. Hong YK, Choi SB, Lee KH et al (2011) The efficacy of portal vein embolization prior to right extended hemihepatectomy for hilar cholangiocellular carcinoma: a retrospective cohort study. Eur J Surg Oncol 37(3):237-244

20. Suzuki H, Iyomasa S, Nimura Y, Yoshida S (1994) Internal biliary drainage, unlike external drainage, does not suppress the regeneration of cholestatic rat liver after partial hepatectomy. Hepatology 20(5):1318-1322

21. Watanapa P (1996) Recovery patterns of liver function after complete and partial surgical biliary decompression. Am J Surg 171(2):230-234

22. Kennedy TJ, Yopp A, Qin Y et al (2009) Role of preoperative biliary drainage of liver remnant prior to extended liver resection for hilar cholangiocarcinoma. HPB (Oxford) 11(5):445-451

23. Takahashi $Y$, Nagino M, Nishio H, Ebata T, Igami T, Nimura $Y$ (2010) Percutaneous transhepatic biliary drainage catheter tract recurrence in cholangiocarcinoma. Br J Surg 97(12):1860-1866

24. Yokoyama Y, Nagino M, Nimura Y (2007) Mechanism of impaired hepatic regeneration in cholestatic liver. J Hepatobiliary Pancreat Surg 14(2):159-166

25. Guiu B, Deschamps F, Aho S et al (2012) Liver/biliary injuries following chemoembolisation of endocrine tumors and hepatocellular carcinoma: lipiodol versus drug-eluting beads. J Hepatol 56(3):609-617

26. Goto Y, Nagino M, Nimura Y (1998) Doppler estimation of portal blood flow after percutaneous transhepatic portal vein embolization. Ann Surg 228(2):209-213
27. Kanda H, Nimura Y, Yasui A, Nakano S, Kumada S, Shionoya S (1997) Recovery of portal blood flow after percutaneous transhepatic biliary drainage in patients with obstructive jaundice. Surg Today 27(2):120-123

28. Ijichi M, Makuuchi M, Imamura H, Takayama T (2001) Portal embolization relieves persistent jaundice after complete biliary drainage. Surgery 130(1):116-118

29. Mizuno S, Nimura Y, Suzuki H, Yoshida S (1996) Portal vein branch occlusion induces cell proliferation of cholestatic rat liver. J Surg Res 60(1):249-257

30. Katoh T, Tanaka M, Nimura Y, Kanai M, Nagino M, Ozawa T (1991) Enhancement of rat liver mitochondrial function by portal branch ligation secures subsequent extended hepatectomy. Biochem Int 24(1):107-116

31. Gall JA, Bhathal PS (1990) A quantitative analysis of the liver following ligation of the common bile duct. Liver 10(2):116-125

32. Rodriguez-Garay EA, Aguero RM, Pisani G, Trbojevich RA, Farroni A, Viglianco RA (1996) Rat model of mild stenosis of the common bile duct. Res Exp Med (Berl) 196(2):105-116

33. Chen MF, Hwang TL, Hung CF (1991) Human liver regeneration after major hepatectomy. A study of liver volume by computed tomography. Ann Surg 213(3):227-229

34. Kele PG, de Boer M, van der Jagt EJ, Lisman T, Porte RJ (2012) Early hepatic regeneration index and completeness of regeneration at 6 months after partial hepatectomy. Br J Surg 99(8):11131119 